Case Report

\title{
Case Report of Synchronous Prostate, Hepatocellular, and Rectal Carcinomas and Review of the Literature
}

\author{
Viktoria Lamprou (D), ${ }^{1}$ Daniel Paramythiotis $\left(\mathrm{D},{ }^{2}\right.$ Dimitrios Giakoustidis $\left(\mathrm{D},{ }^{3}\right.$ \\ Anestis Karakatsanis $\mathbb{D}^{2},{ }^{2}$ Athanasios Astreinidis $\mathbb{D}^{1},{ }^{1}$ Moysis Moysidis $\mathbb{D}^{\circ},{ }^{2}$ \\ Antonios Mihalopoulos $\mathbb{D}^{2},{ }^{2}$ and Stefanos Finitsis $\mathbb{D}^{1}$ \\ ${ }^{1}$ Department of Interventional Radiology, University General Hospital of Thessaloniki AHEPA, Thessaloniki 546 21, Greece
${ }^{2} 1$ st Propaedeutic Surgery Department, University General Hospital of Thessaloniki AHEPA, Thessaloniki 546 21, Greece
${ }^{3} 1$ st Surgery Department, Aristotle University of Thessaloniki, General Hospital Papageorgiou, Thessaloniki 56403, Greece
}

Correspondence should be addressed to Viktoria Lamprou; victorialambrou@gmail.com

Received 10 October 2019; Accepted 10 January 2020; Published 22 January 2020

Academic Editor: Christophoros Foroulis

Copyright (c) 2020 Viktoria Lamprou et al. This is an open access article distributed under the Creative Commons Attribution License, which permits unrestricted use, distribution, and reproduction in any medium, provided the original work is properly cited.

\begin{abstract}
Synchronous occurrence of three histopathologically distinct malignant tumors is a rare event, and there are no definitive guidelines about the optimal treatment of these patients. We report a case of synchronous prostate, hepatocellular, and rectal carcinomas and discuss our therapeutic strategy that resulted in excellent clinical results.
\end{abstract}

\section{Introduction}

Synchronous malignancies are defined as tumors diagnosed within two to six months of the initial diagnosis of a primary tumor and occur in $0,002 \%$ to $1,96 \%$ of cancer patients $[1,2]$. This frequency is increasing despite a general decline in cancer deaths since 1991 because of the increased lifespan of the general population secondary to proper screening, earlier diagnosis, improvements in treatment, and surveillance of patients [3]. Management of synchronous cancers is challenging [4]. We present a patient with synchronous liver, colorectal, and prostate cancers treated by a multidisciplinary approach with an excellent clinical outcome.

\section{Case Presentation}

A 67-year-old man presented with a history of change of bowel habits, blood in stool, and weight loss. His past medical history included only tobacco and alcohol use. According to his family history, two of his first-degree relatives had developed colonic cancer. A colonoscopy revealed the presence of a $4 \mathrm{~cm}$ mass approximately $10 \mathrm{~cm}$ from the anal verge and was confirmed to be a well-differentiated colon adenocarcinoma at a biopsy. Laboratory tests showed elevated serum carcinoembryonic antigen (CEA) $(8,07 \mathrm{ng} / \mathrm{ml})$, elevated serum prostate-specific antigen (PSA) $(17,87 \mathrm{ng} / \mathrm{ml})$, and elevated $\alpha$-FP $(17,9 \mathrm{ng} / \mathrm{ml})$. The patient was seronegative for HBsAg and anti-HCV (IgM and IgG). His liver function tests were within normal limits. The abdominal MRI showed the presence of an $11 \times 10,5 \times 10,5 \mathrm{~cm}$ mass in the right hepatic lobe, with radiologic features consistent with a primary liver lesion (Figures 1 and 2). The CTguided liver biopsy revealed a well-differentiated hepatocellular carcinoma. Additionally, because of the elevated PSA values, a prostate biopsy was performed. Seven core biopsies were taken from the left prostatic lobe and eight from the right lobe, and all of them were positive for prostatic adenocarcinoma (Gleason score $3+4=7 / 10$ ).

Given the patient's good performance status, surgical resection for the hepatocellular carcinoma was considered. However, because of the small liver remnant, it was decided to first perform transarterial chemoembolization, to partially control the hepatocellular carcinoma (Figure 3). Two weeks later, the rectal carcinoma was treated by low anterior 


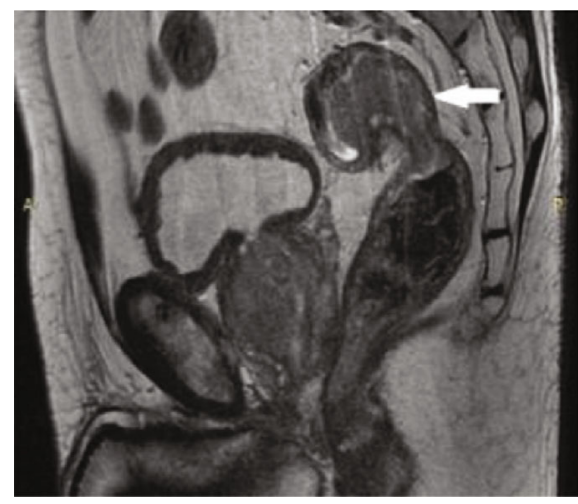

(a)

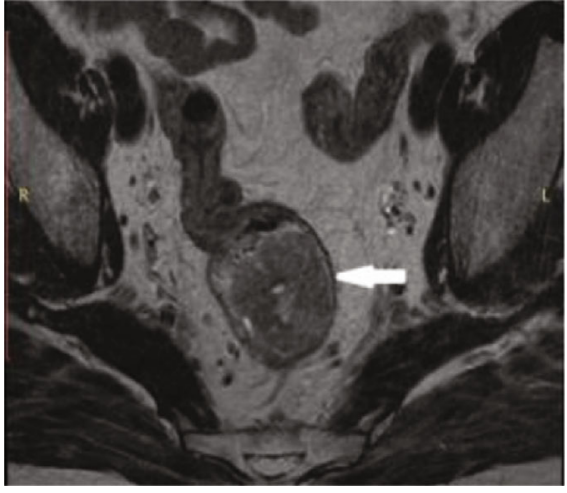

(b)

FIGURE 1: Sagittal (a) and axial (b) reconstruction of a T2-weighted MRI showing the rectal tumor (arrow).

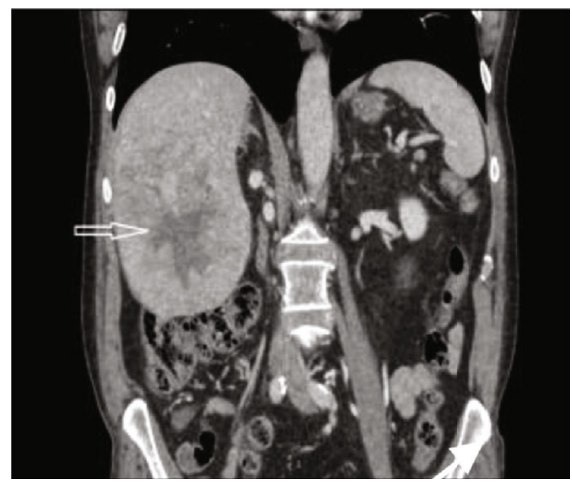

(a)

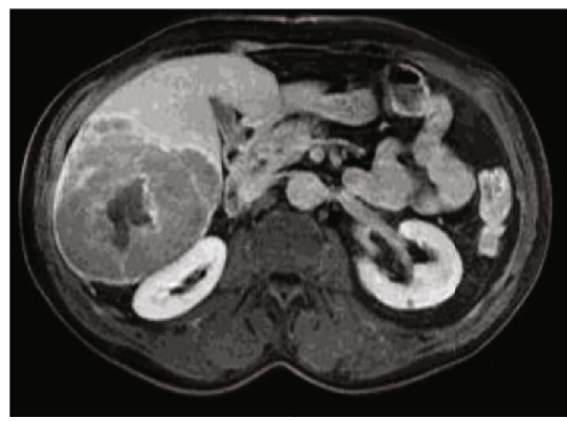

(c)

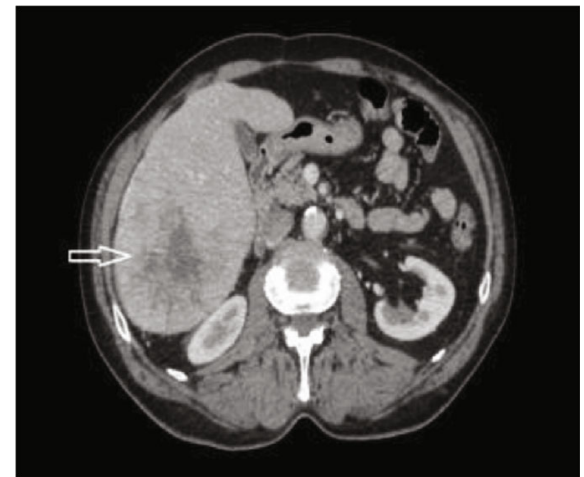

(b)

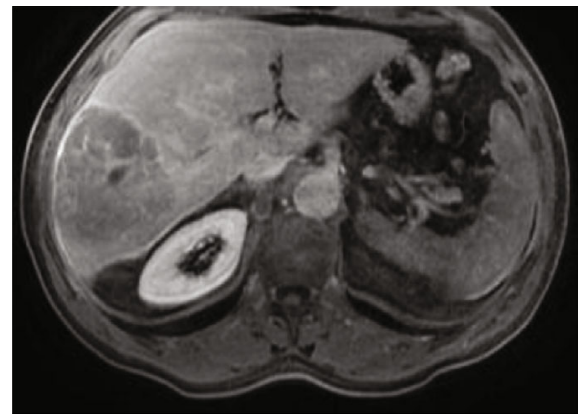

(d)

Figure 2: Coronal (a) and axial (b) CT scan images showing a large ( $>10 \mathrm{~cm})$ inhomogeneously enhancing liver mass with a central scar occupying liver segments V, VI, and VII (arrow). (c, d) Axial post contrast MRI image demonstrating heterogeneously enhancing liver mass with washout and central nonenhancing component, features mostly consistent with atypical HCC.

resection (Figure 4). After six weeks, right portal vein embolization was performed to allow for hypertrophy of the liver remnant (Figure 5). Finally, one month later, the hepatocellular carcinoma was treated by right hepatectomy (Figures 4 and 6). Active surveillance was adopted for the prostate cancer.

Histopathological evaluation of the rectal specimen showed a moderately differentiated adenocarcinoma Stage IIA (T3NOM0) and a well-differentiated hepatocellular adenocarcinoma Stage IIIA (T3NOM0). Recovery was uneventful, and the patient remained disease-free up until the 2-year follow-up when he died of acute myocardial infarction.

\section{Discussion}

According to the Surveillance Epidemiology and End Results (SEER) project and the International Association of Cancer Registries and International Agency for Research on Cancer (IACR/IARC), synchronous multiple primaries are defined 


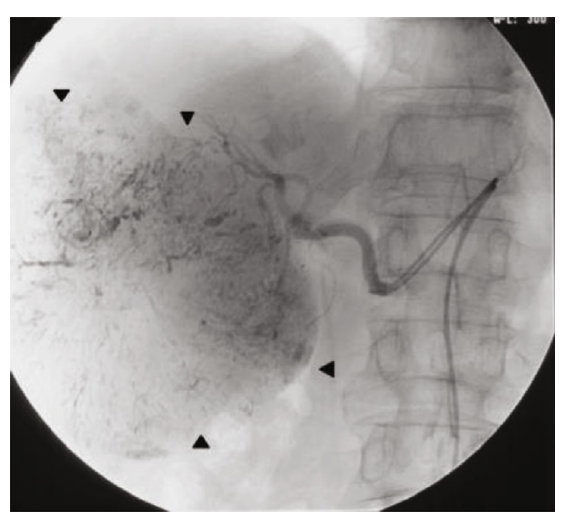

(a)

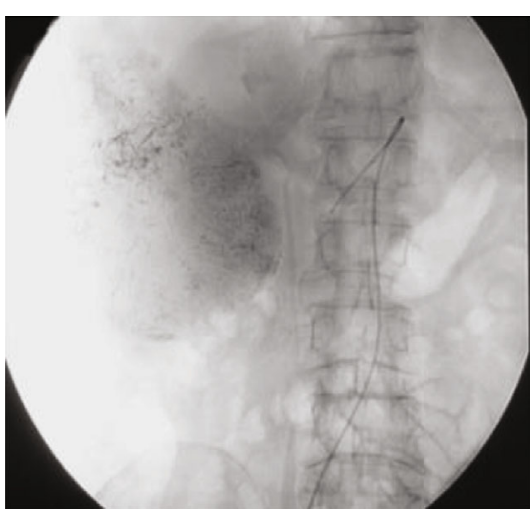

(b)

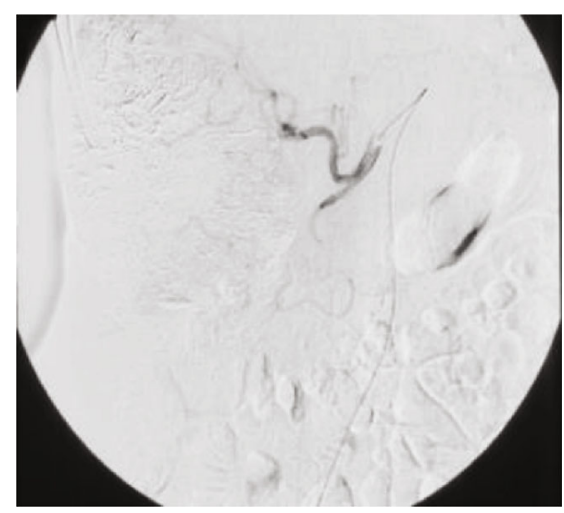

(c)

Figure 3: (a) Hepatic arteriogram shows a large tumor staining in the right hepatic lobe (arrowheads). (b, c) Arteriogram after chemoembolization of the tumor, and its feeding branch shows no residual tumor enhancement.

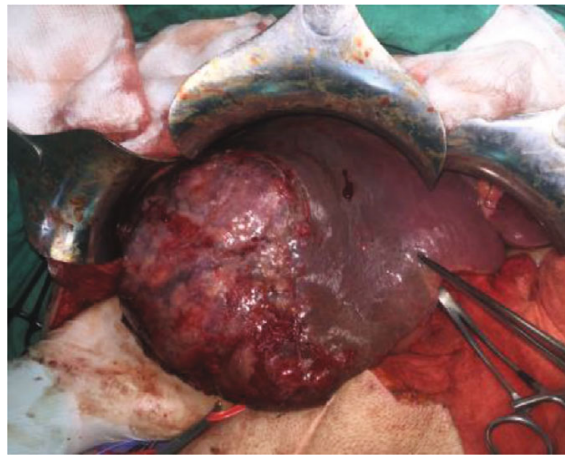

(a)

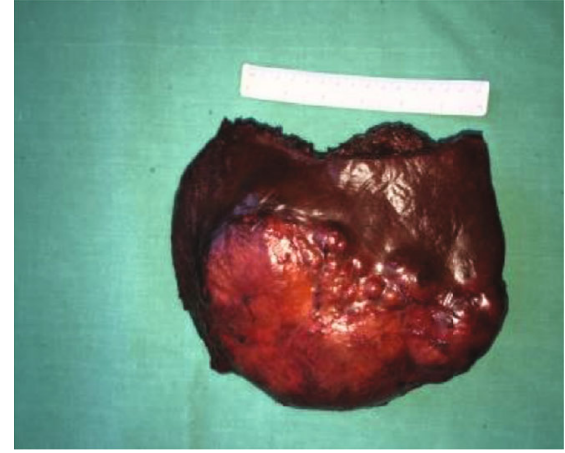

(b)

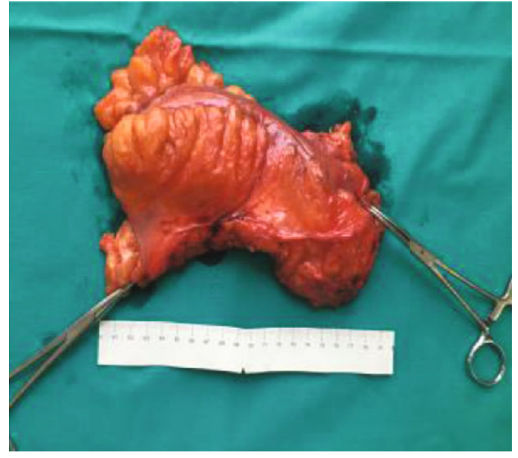

(c)

FIGURE 4: Intraoperative photo of HCC (a). Surgical specimen of the liver (b) and the rectosigmoid colon (c).

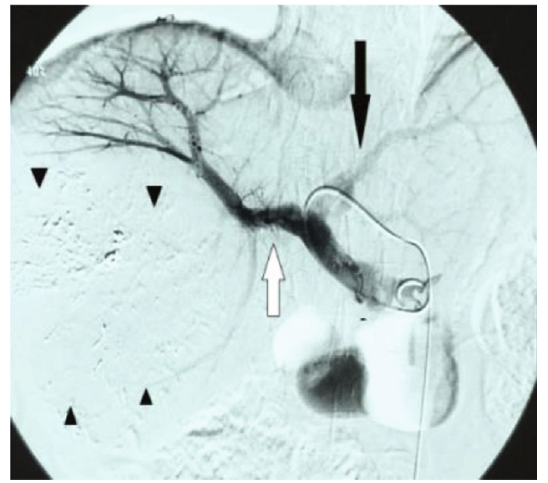

(a)

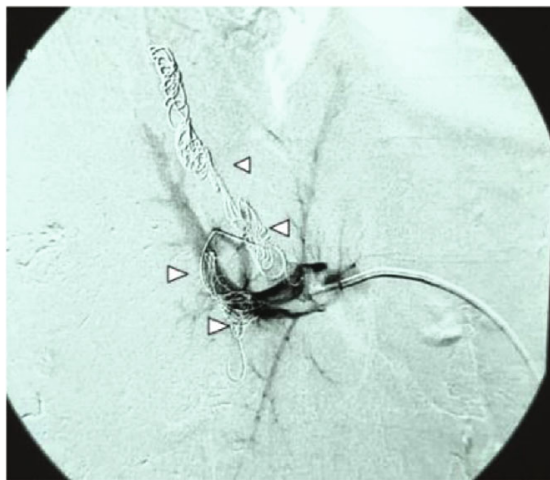

(b)

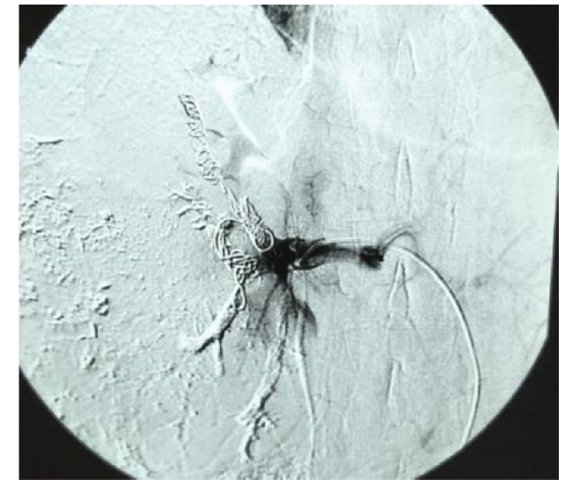

(c)

Figure 5: (a) Portal venogram showing the right portal vein (white arrow), the left portal vein (black arrow), and the HCC (black arrowheads). (b) Coils in the anterior and posterior branches of the right portal vein (white arrowheads). (c) Final portal venogram shows occlusion of the right portal vein branches.

as two or more independent primaries diagnosed within two to six months of the initial diagnosis $[4,5]$. Factors associated with increased risk of developing multiple primary malignancies include environmental exposures, genetic factors (family cancer syndromes and other genetic susceptibility factors), lifestyle (tobacco and alcohol consumption), immunodeficiency syndromes (either acquired or inherited), and carcinogenic effects of cancer treatment [6]. 


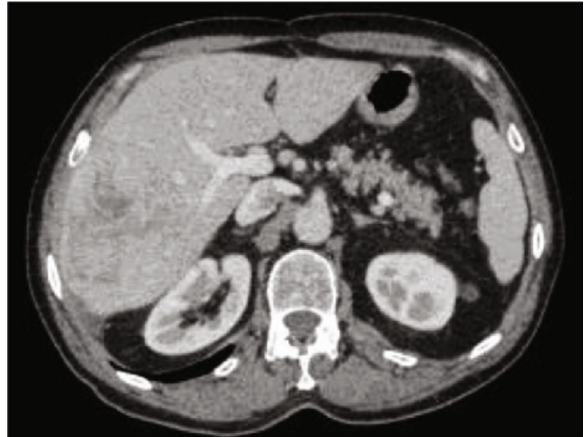

(a)

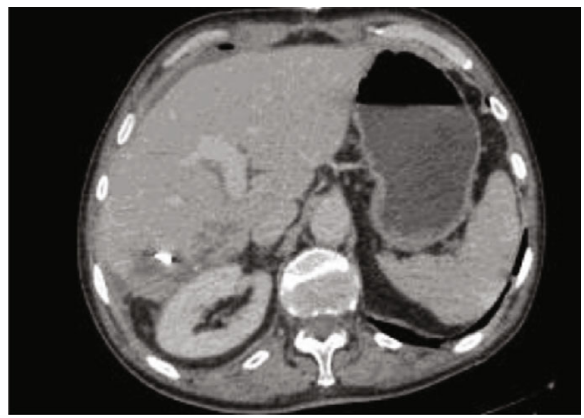

(c)

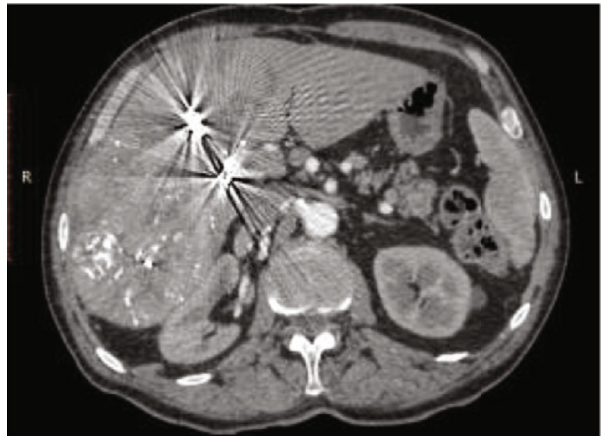

(b)

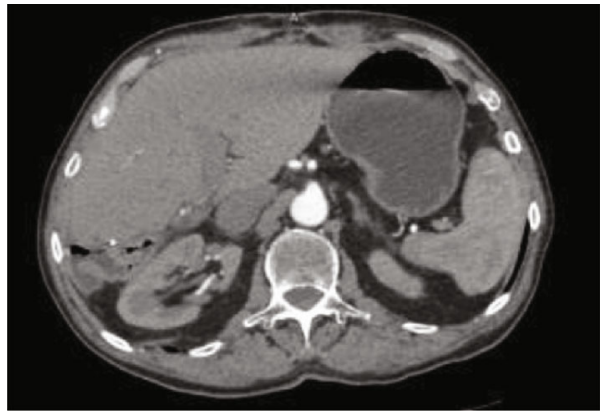

(d)

Figure 6: (a) CT scan before TACE and PVE. (b) One month after PVE and three months after TACE, anticipated hypertrophy of LLL is observed. On CT scan, one month (c) and three months (d) after liver resection, there is no evidence of HCC recurrence. Significant hypertrophy of the liver remnant is noted.

Male genital cancers and urinary tract cancers are the most widespread combination of synchronous multiple malignancies, followed by colorectal and noncolorectal digestive tract cancers [2]. Specifically, a synchronous occurrence of rectal and prostate cancers has been described approximately in $0,1 \%-30,5 \%$ of cancer patients $[7,8]$. In HCC patients, prostate and rectal tumors coexist in $0,05 \%$ and $0,4 \%$ of cases, respectively $[9,10]$. Triple synchronous primary tumors involving hepatocellular, rectum, and prostate cancers are a rare occurrence with few cases reported in the English literature (Table 1).

During pretreatment work-up of cancer patients, the diagnosis of a second mass raises the possibility either of metastasis or of a synchronous primary malignant tumor. When multiple tumors are pathologically confirmed, the therapeutic approach is decided after staging each tumor independently. Moreover, the patient's age, life expectancy, and comorbidities as well as the tumor's biological behavior should be considered. Treatment options include curative surgical resection of each tumor, when possible in a single setting, radiation, or chemoradiation [26-28]. As there exist no systematic and authoritative treatment guidelines for the treatment of synchronous primary malignancies, the aim is to cover all primaries without negatively affecting the overall patient's outcome.

Especially for triple synchronous carcinomas, treatment strategies remain poorly defined. Several studies have failed to show a significant impact of a second extra hepatic malignancy on the survival of patients with HCC, suggesting that aggressive treatment of $\mathrm{HCC}$ by curative resection is an inde- pendent prognostic factor associated with a good outcome $[29,30]$. In the present case, we prioritized the cure of the hepatocellular carcinoma independently of two other malignancies. However, the successful implementation of hepatectomy represented a therapeutic challenge due to the large size of the tumor and the small functional liver remnant. We opted for sequential TACE and PVE of the hepatocellular carcinoma as this strategy is associated with longer recurrence-free survival $[31,32]$. TACE allowed for local control of the hepatocellular carcinoma during which the rectal carcinoma was resected, and PVE was performed allowing eventual radical HCC excision. Taking into account the presence of two other synchronous malignancies and patient's preferences, active therapy of prostate cancer was withheld, and active surveillance was adopted. Recent guidelines for the follow-up of HCC after liver resection recommend dynamic CT/MRI studies every 3 months during the first year and every 6 months thereafter [33]. Follow-up recommendations for rectal carcinoma include rectosigmoidoscopy and CEA testing every 6 months for 5 years and abdominal and chest CT scan annually for 3 years [34]. Our patient kept the above-mentioned follow-up protocol for six months and opted for a simple clinical follow-up for two years, during which he remained in an excellent clinical condition.

\section{Conclusion}

Our case highlights the value of targeted curative therapy of synchronous malignancies in a framework of a patient- 


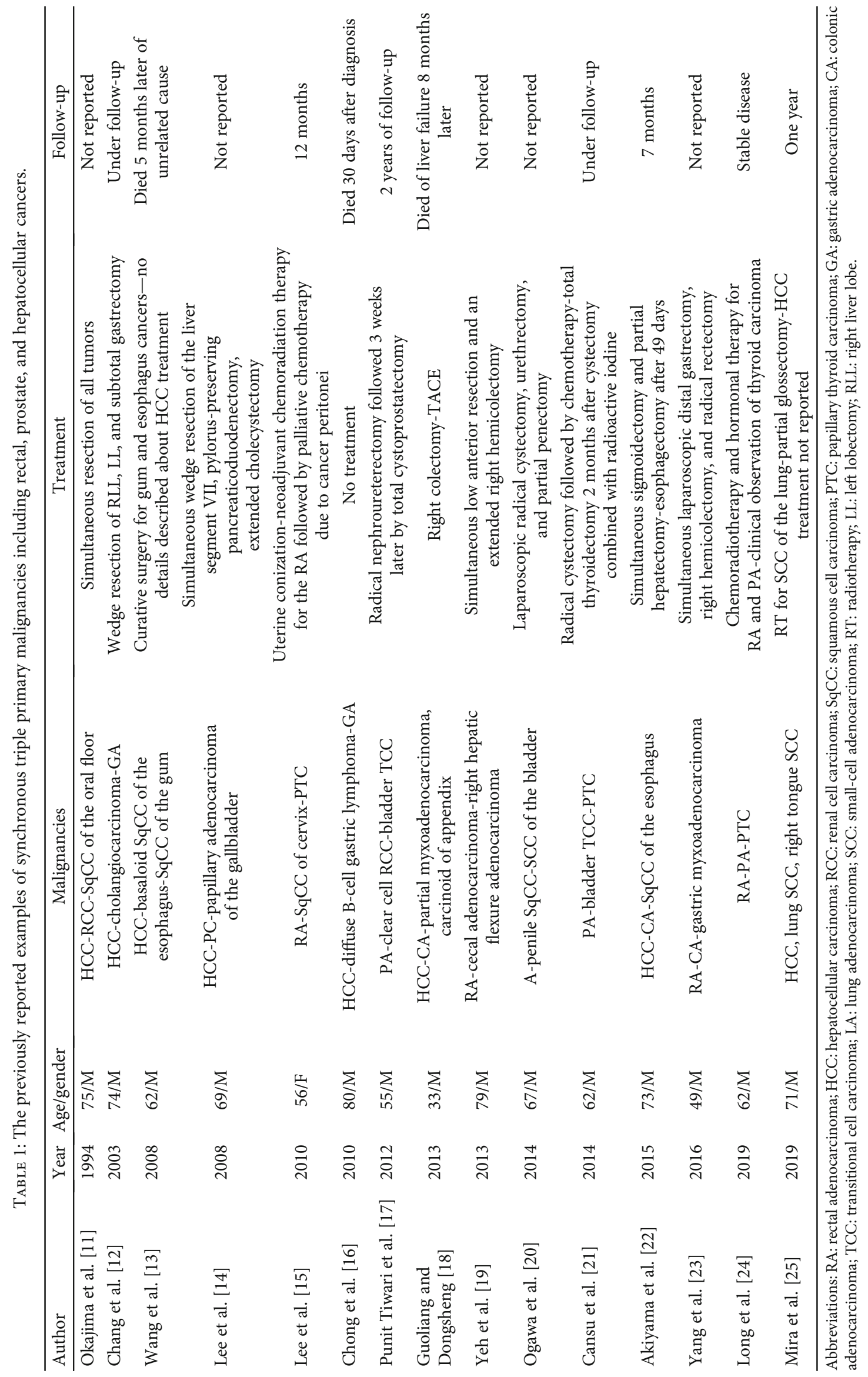


centered multidisciplinary approach. We achieved an excellent result with curative therapy of two of the three synchronous lesions and opted for conservative management of the prostate cancer.

\section{Conflicts of Interest}

The authors declare that there is no conflict of interest regarding the publication of this paper.

\section{References}

[1] A. Irimie, P. Achimas-Cadariu, C. Burz, and E. Puscas, "Multiple primary malignancies - epidemiological analysis at a single tertiary institution," Journal of Gastrointestinal and Liver Diseases, vol. 19, no. 1, pp. 69-73, 2010.

[2] A. Y. Lin, T. Lu, and S. L. Gomez, "Synchronous cancers: an analysis of SEER registry from 1973 to 2003," Journal of Clinical Oncology, vol. 28, article e12018, 2010.

[3] C. Facts, American Cancer Society. Cancer Facts \& Figures 2018, American Cancer Society, Atlanta, 2018.

[4] A. Vogt, S. Schmid, K. Heinimann et al., "Multiple primary tumours: challenges and approaches, a review," ESMO Open, vol. 2, no. 2, article e000172, pp. 1-11, 2017.

[5] C. Johnson, The Seer Program Coding and Staging Manual 2004, Revision 1 Cancer Statistics Branch, Cancer Statistics Branch, Surveillance Research Program, Division of Cancer Control and Population Sciences, National Cancer Institute, National Institutes of Health, Public Health Service, U.S. Department of Health and Human Services, Bethesda, MD, USA, 2006.

[6] C. Facts, American Cancer Society. Cancer Facts \& Figures 2009, American Cancer Society, Atlanta, 2009.

[7] M. Sturludóttir, A. Martling, S. Carlsson, and L. Blomqvist, "Synchronous rectal and prostate cancer - the impact of MRI on incidence and imaging findings," European Journal of Radiology, vol. 84, no. 4, pp. 563-567, 2015.

[8] K. Ung, J. Brierley, A. J. Bayley et al., "Treatment dilemmas for synchronous and metachronous prostate and rectal cancers," International Journal of Radiation Oncology•Biology•Physics, vol. 90, no. 1, article S385, 2014.

[9] W. Xu, W. Liao, P. Ge et al., "Multiple primary malignancies in patients with hepatocellular carcinoma," Medicine, vol. 95, no. 17, pp. e3491-e3497, 2016.

[10] K. M. Kee, J. H. Wang, C. C. Wang, Y. F. Cheng, and S. N. Lu, "Hepatocellular carcinoma associated with extra-hepatic primary malignancy: its secular change, clinical manifestations and survival," Scientific Reports, vol. 6, no. 1, pp. 1-9, 2016.

[11] E. Okajima, S. Ozono, J. Nagayoshi et al., "A case report of synchronous triple cancer resected simultaneously," Japanese Journal of Clinical Oncology, vol. 24, no. 3, pp. 166-170, 1994.

[12] J. Y. Chang, B. H. Kim, S. W. Hong, Y. W. Kim, and J. H. Oh, "A case report of synchronous double primary liver cancers combined with early gastric cancer," The Korean Journal of Internal Medicine, vol. 18, no. 2, pp. 115-118, 2003.

[13] P. Wang, G. Zhang, and H. Shen, "Multiple synchronous primary malignancies induced by benzene exposure: a case report," Journal of Occupational Medicine and Toxicology, vol. 4, no. 1, pp. 1-4, 2009.
[14] J. Lee, B. Kim, H. Wang, and M. Kim, "Synchronous triple primary cancers - liver, gallbladder and pancreas," Korean Journal of Hepatobiliary Pancreas, vol. 12, no. 4, pp. 294-297, 2008.

[15] J. S. Lee, W. Moon, S. J. Park et al., “Triple synchronous primary cancers of rectum, thyroid, and uterine cervix detected during the workup for hematochezia," Internal Medicine, vol. 49, no. 16, pp. 1745-1747, 2010.

[16] V. H. Chong, A. Idros, and P. U. Telisinghe, "Triple synchronous gastrointestinal malignancies: a rare occurrence," Singapore Medical Journal, vol. 51, no. 10, pp. 10-12, 2010.

[17] P. Tiwari, A. Tripathi, P. Bansal, M. Vijay, A. Gupta, and A. K. Kundu, "Synchronous primary cancers of urinary bladder and kidney and prostate," Saudi Journal of Kidney Diseases and Transplantation, vol. 23, no. 4, pp. 786-789, 2012.

[18] S. Guoliang and H. Dongsheng, "Triple synchronous malignant tumors of colon, appendix and liver: a case report with literature review," Pakistan Journal of Medical Sciences, vol. 29, no. 1, pp. 237-238, 2012.

[19] C. C. Yeh, S. C. Hsi, C. P. Chuu, and Y. H. Kao, "Synchronous triple carcinoma of the colon and rectum," World Journal of Surgical Oncology, vol. 11, no. 1, p. 66, 2013.

[20] S. Ogawa, T. Yasui, K. Taguchi, Y. Umemoto, Y. Kojima, and K. Kohri, "The probability of involvement of human papillomavirus in the carcinogenesis of bladder small cell carcinoma, prostatic ductal adenocarcinoma, and penile squamous cell carcinoma: a case report," BMC Research Notes, vol. 7, no. 1, pp. 1-5, 2014.

[21] G. B. Cansu, G. A. G. Ocak, and R. Sari, "Triple synchronous primary cancers of thyroid, bladder and prostate: a case report," Kuwait Medical Journal, vol. 46, no. 1, pp. 62-64, 2014.

[22] Y. Akiyama, T. Iwaya, M. Konosu et al., "Curative two-stage resection for synchronous triple cancers of the esophagus, colon, and liver: report of a case," International Journal of Surgery Case Reports, vol. 13, pp. 1-4, 2015.

[23] L. Yang, D. Zhang, F. Li, and X. Ma, "Simultaneous laparoscopic distal gastrectomy (uncut Roux-en-Y anastomosis), right hemi-colectomy and radical rectectomy (Dixon) in a synchronous triple primary stomach, colon and rectal cancers patient," Journal of Visualized Surgery, vol. 2, p. 101, 2016.

[24] Y. Long, W. Liu, Y. Dai et al., "Synchronous primary carcinomas of the prostate, thyroid and rectum: case report and review of the literature," Translational Cancer Research, vol. 8, no. 3, pp. 1006-1011, 2019.

[25] R. R. Mira, A. Bial, K. H. Campbell, S. Ali, S. Abdulameer, and M. J. Gorbien, "Metachronous and synchronous multiple primary carcinomas in an elderly," Journal of Medical Cases, vol. 10, no. 1, pp. 8-13, 2019.

[26] Y. Zhang, Y. Ge, X. Wu, and S. Liu, "Clinical treatment of advanced synchronous triple primary malignancies: comprehensive treatment based on targeted therapy," OncoTargets and Therapy, vol. 12, pp. 2421-2430, 2019.

[27] H. Wu, Q. Lu, N. Li et al., "Clinical retrospective analysis of 20 cases with digestive tract multiple primary malignant neoplasms," International Journal of Clinical and Experimental Medicine, vol. 10, no. 11, pp. 15498-15503, 2017.

[28] T. Chowdary, S. Sivaraj, G. Rao, and S. Thirunavukkarasu, "Dual malignancies: do they have a worse prognosis than their individual counterparts," Archives of International Surgery, vol. 5, no. 1, p. 29, 2015.

[29] Q. A. Zeng, J. Qiu, R. Zou et al., “Clinical features and outcome of multiple primary malignancies involving hepatocellular 
carcinoma: a long-term follow-up study," BMC Cancer, vol. 12 , no. $1,2012$.

[30] M. L. Yeh, C. I. Huang, C. F. Huang et al., "The impact of an additional extra-hepatic primary malignancy on the outcomes of patients with hepatocellular carcinoma," PLoS One, vol. 12, no. 9, article e0184878, 2017.

[31] M. Ronot, F. Cauchy, B. Gregoli et al., "Sequential transarterial chemoembolization and portal vein embolization before resection is a valid oncological strategy for unilobar hepatocellular carcinoma regardless of the tumor burden," $H P B$, vol. 18, no. 8, pp. 684-690, 2016.

[32] S. Ogata, J. Belghiti, O. Farges, D. Varma, A. Sibert, and V. Vilgrain, "Sequential arterial and portal vein embolizations before right hepatectomy in patients with cirrhosis and hepatocellular carcinoma," The British Journal of Surgery, vol. 93, no. 9, pp. 1091-1098, 2006.

[33] A. Vogel, A. Cervantes, I. Chau et al., "Hepatocellular carcinoma: ESMO Clinical Practice Guidelines for diagnosis, treatment and follow-up," Annals of Oncology, vol. 29, pp. iv238iv255, 2018.

[34] J. A. Meyerhardt, P. B. Mangu, P. J. Flynn et al., "Follow-up care, surveillance protocol, and secondary prevention measures for survivors of colorectal cancer: American Society of Clinical Oncology clinical practice guideline endorsement," Journal of Clinical Oncology, vol. 31, no. 35, pp. 4465-4470, 2013. 


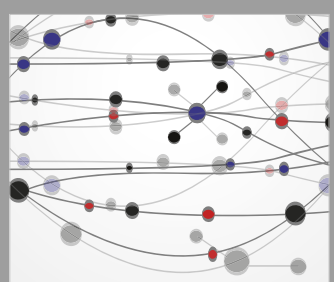

The Scientific World Journal
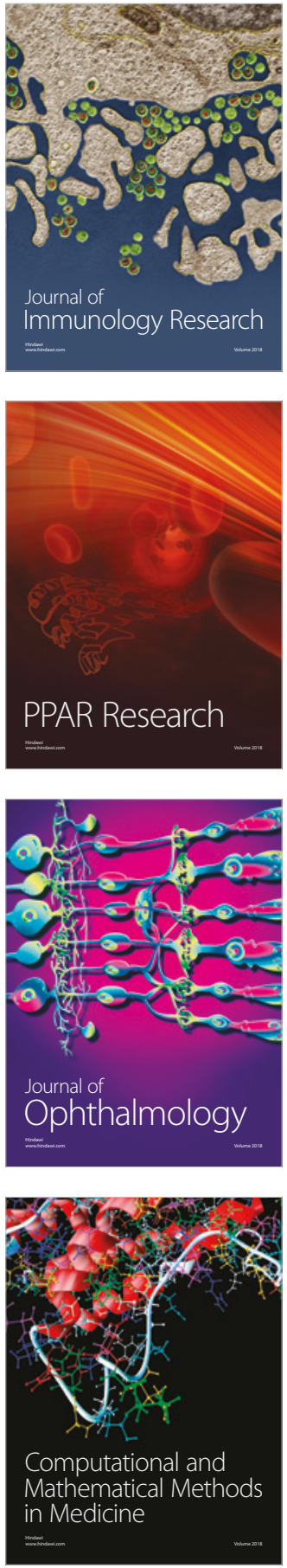

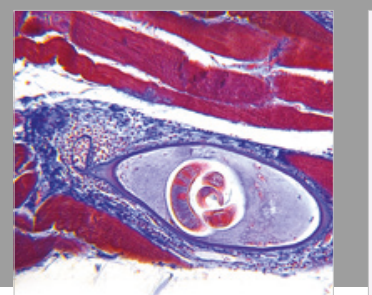

Gastroenterology Research and Practice

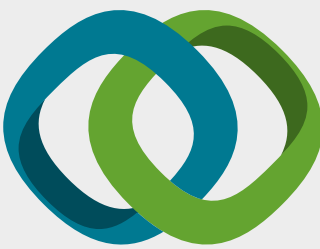

\section{Hindawi}

Submit your manuscripts at

www.hindawi.com
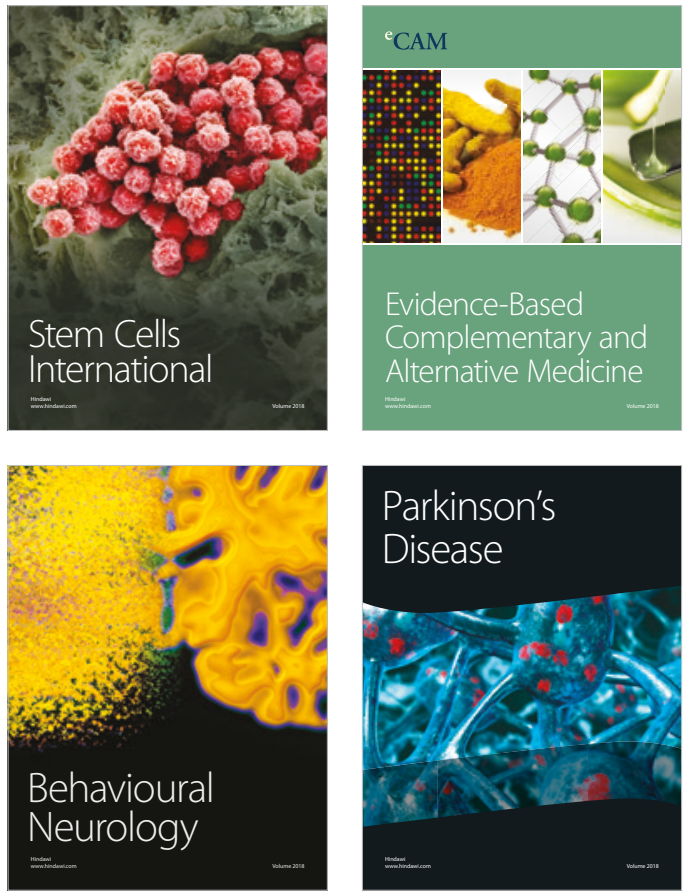

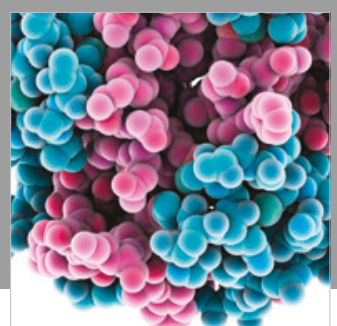

ournal of

Diabetes Research

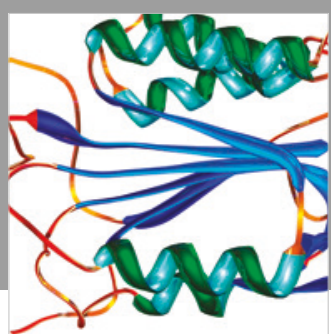

Disease Markers
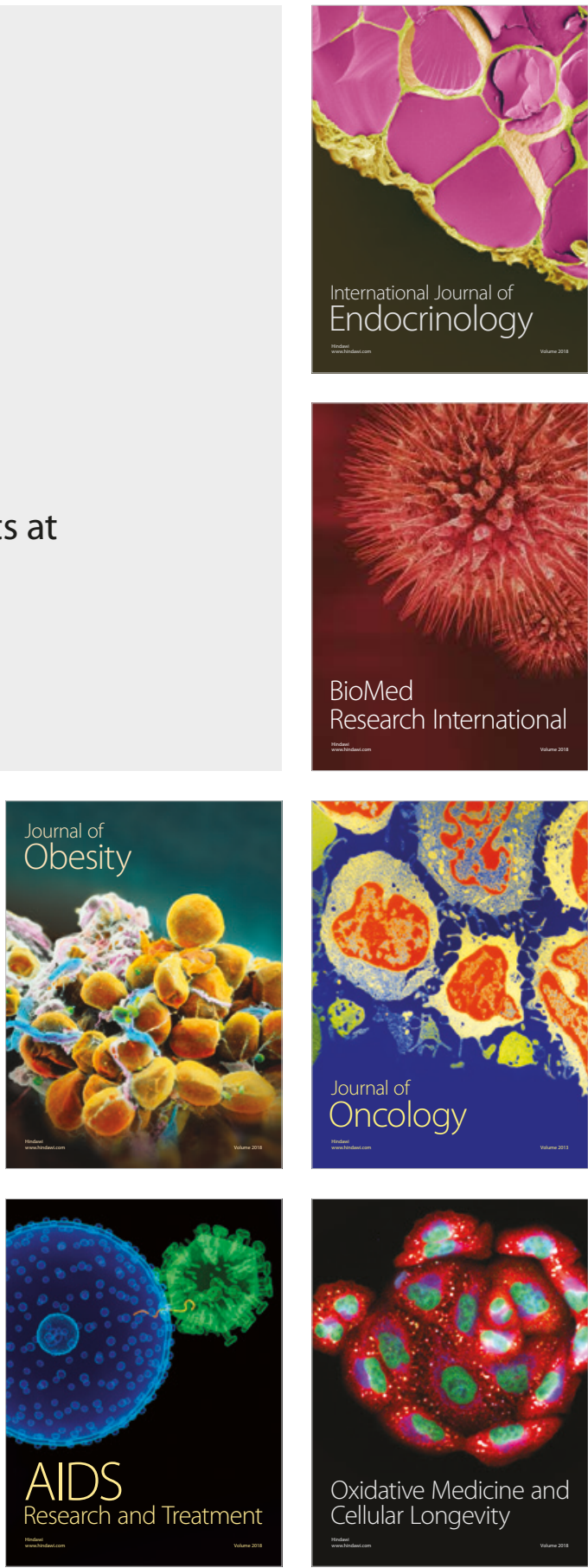